\title{
A Supersymmetric Extension of the Kadomtsev-Petviashvili Hierarchy
}

\author{
Yu. I. Manin ${ }^{1}$ and A. O. Radul ${ }^{2}$ \\ 1 Steklov Mathematical Institute, Moscow, USSR \\ 2 Moscow State University, Moscow, USSR
}

\begin{abstract}
An extension of the Kadomtsev-Petviashvili hierarchy by odd variables is given. Conservation laws and formal integrability are proved.
\end{abstract}

\section{Introduction}

It is well known that integrable systems usually admit a natural extension by odd variables, see e.g. [1-4].

In the articles $[5,6]$ an infinite hierarchy of nonlinear differential equations was studied with the property that many known completely integrable systems can be obtained from this hierarchy by various reductions. It is called the KadomtsevPetviashvili hierarchy and it can be succinctly written in the following way. Let $x$ be a space variable and $t_{1}, t_{2}, \ldots$ an infinite system of time variables. Denote by $u_{-1}, u_{-2}, \ldots$ an infinite set of functions depending on $x, t_{1}, t_{2}, \ldots$. Set $\partial=\partial / \partial x$ and introduce a formal pseudo-differential operator $L=\partial+\sum_{i=1}^{\infty} u_{-i} \partial^{-i}$. The
Kadomtsev-Petviashvili (KP) hierarchy is

$$
\partial_{i} L=\left[L_{+}^{i}, L\right], \quad \partial_{i}=\partial / \partial t_{i},
$$

where $A_{+}$is the differential part of an operator $A$. If in addition $L^{2}=\left(L^{2}\right)_{+}$, Eq. (1) becomes the $i^{\text {th }}$ equation of the Korteweg-de Vries hierarchy (KdV itself corresponding to $i=3$ ).

The objective of this note is to introduce a new system of equations for an infinite set of even and odd functions, depending on an even-odd pair of space variables $(x, \xi)$ and even-odd times $\left(\tau_{1}, t_{2}, \tau_{3}, t_{4}, \ldots\right)$. We shall call this system the supersymmetric Kadomtsev-Petviashvili (SKP) hierarchy, since KP is its natural reduction. We shall show that SKP shares the standard properties of "completely integrable" systems, e.g. has infinitely many conservation laws, is formally solvable by the Zakharov-Shabat method, and can be reduced to Lax- and Gelfand-Dikii (Dickey) equations. In a subsequent publication we hope to discuss the solitons and algebraic type solutions of SKP, as well as the transformation groups for this hierarchy. 
Section 1 contains definitions and statements of principal results. The proofs are given in Sect. 2 .

\section{Definitions and Results}

\subsection{Pseudodifferential Operators on the $1 \mid 1$ Line}

The reader may consult [7 or 8] for the background in superalgebra and supergeometry.

We fix an even variable $x$ and odd one $\xi$; in general we set $\tilde{X}=0$ (respectively 1), if $X$ is even (respectively odd). Set $\theta=\partial / \partial \xi+\xi \partial / \partial x$. Then $\theta^{2}=\frac{1}{2}[\theta, \theta]=\partial$. (We recall that the supercommutator is defined by $[X, Y]=X Y-(-1)^{\tilde{X} \tilde{Y}} Y X$.)

Let $B$ be a $\mathbb{Z}_{2}$-graded ring, on which $\theta$ acts as an odd superderivation, $\tilde{\theta b}=\tilde{b}+1, \theta(b c)=(\theta b) c+(-1)^{\hat{b}} b \theta c$. We shall write $\theta^{i} b=b^{[i]}$. The ring of formal pseudodifferential operators $B\left(\left(\theta^{-1}\right)\right)$ consists of the formal series $L=\sum_{i \leq m} b_{i} \theta^{i}$, $b_{i} \in B$. It is $\mathbb{Z}_{2}$-graded by $\tilde{b}^{i}=\tilde{b}+\tilde{i}$. We set $L_{+}=\sum_{0 \leqq i \leqq m} b_{i} \theta^{i}, L_{-}=\sum_{i \leqq-1} b_{i} \theta^{i}$.

The multiplication in $B\left(\left(\theta^{-1}\right)\right)$ is best described by means of "superbinomial coefficients" $\left[\begin{array}{c}m \\ k\end{array}\right], k, m \in \mathbb{Z}$. First recall the ordinary coefficients: $\left(\begin{array}{c}m \\ k\end{array}\right)=0$ for $k>m$, 1 for $k=m,(k+1) \ldots m /(m-k)$ ! for $k<m$. Now we set

$$
\left[\begin{array}{l}
j \\
k
\end{array}\right]= \begin{cases}0 & \text { for } \quad k>j \quad \text { and for }(j, k) \equiv(0,1) \bmod 2 \\
\left(\begin{array}{c}
{[j / 2]} \\
{[k / 2]}
\end{array}\right) & \text { for } k \leqq j, \quad(j, k) \neq(0,1) \bmod 2,\end{cases}
$$

Then we define

$$
\begin{gathered}
\theta^{j} \circ a=\sum_{k \leqq j}\left[\begin{array}{l}
j \\
k
\end{array}\right](-1)^{\tilde{a} k} a^{[j-k]} \theta^{k}, \\
\sum b_{j} \theta^{j} \circ \sum a_{\ell} \theta^{\ell}=\sum_{j, k, \ell}\left[\begin{array}{l}
j \\
k
\end{array}\right](-1)^{\tilde{a}_{t} k} b_{j} a_{\ell}^{[j-k]} \theta^{k+\ell}
\end{gathered}
$$

[we sometimes use $\circ$ for multiplication in $B\left(\left(\theta^{-1}\right)\right)$ to distinguish e.g. $\theta^{j} \circ a$ from $\theta^{j} a$ $\left.=a^{[j]}\right]$.

\subsection{Flows with Even and Odd Times}

The evolution in KP (1) is given by specifying the derivations with respect to the commuting family of the flows $\partial_{i}$. The evolution in SKP is defined with respect to a non-abelian Lie superalgebra of flows $\theta_{i}, i \geqq 1, \tilde{\theta_{i}}=\tilde{i}$ with the commutation relations

$$
\left[\theta_{2 i}, \theta_{2 j}\right]=0, \quad\left[\theta_{2 i}, \theta_{2 j-1}\right]=0, \quad\left[\theta_{2 i-1} \theta_{2 j-1}\right]=2 \theta_{2 i+2 j-2} .
$$


The choice of (5) is motivated by the fact that $\theta_{i} \rightarrow \theta^{i}$ is a representation of (5), in the same manner as $\partial_{i} \rightarrow \partial^{i}$ is a representation of the abelian Lie algebra of KP.

Let us now introduce the even and odd time variables $\left(\tau_{1}, t_{2}, \tau_{3}, \ldots\right)$, and the following representation of (5) which we shall use in what follows:

$$
\theta_{2 i}=\partial / \partial t_{2 i}, \quad \theta_{2 i-1}=\partial / \partial \tau_{2 i-1}+\sum_{j=1}^{\infty} \tau_{2 j-1} \partial / \partial t_{2 i+2 j-2}
$$

With minor changes we could also use a more general representation

$$
\theta_{2 i-1}=\partial / \partial \tau_{2 i-1}+\sum_{j=1}^{\infty} T_{2 j-1}^{2 i}(\tau) \partial / \partial t_{2 j}
$$

where $T$ are some odd polynomials with constant coefficients such that

$$
\partial / \partial \tau_{2 i-1} T_{2 k-1}^{2 \ell}+\partial / \partial \tau_{2 k-1} T_{2 i-1}^{2 \ell}=2 \delta_{\ell, i+k-1} .
$$

\subsection{The Definition of the SKP Hierarchy}

Let us assume now that the Lie superalgebra (5) is represented by superderivations of the ring $B$, supercommuting with $\theta$. This means that the elements of $B$ can be informally considered as functions of $x, \xi, \tau_{i}, t_{j}$. We shall extend the action of $\theta_{i}$ to $B\left(\left(\theta^{-1}\right)\right)$ in the obvious way.

Now we shall consider an odd pseudodifferential operator

$$
\Lambda=\theta+\sum_{i=0}^{\infty} u_{-i} \theta^{+i}, \quad \tilde{u}_{-i}=\tilde{i}+1, \quad u_{i} \in B
$$

and we shall call the SKP hierarchy the following system:

$$
\left.\begin{array}{l}
\theta_{2 i} \Lambda=-\left[\Lambda^{2 i}, \Lambda\right] \\
\theta_{2 i-1} \Lambda=-\left[\Lambda^{2 i-1}+, \Lambda\right]+2 \Lambda^{2 i} .
\end{array}\right\}
$$

To explain the appearance of $-2 \Lambda^{2 i}$ in (7), recall that the ordinary KP (1) can be written also in the form $\partial_{j} L=-\left[L_{-}^{j}, L\right]$ since $\left[L^{j}+L_{--}^{j}, L\right]=\left[L^{j}, L\right]=0$. In our case $\left[\Lambda^{j}{ }_{+}+\Lambda^{j}{ }_{-}, \Lambda\right]=\left[\Lambda^{j}, \Lambda\right]=0$ for even $j$, but it is $2 \Lambda^{j+1}$ for odd $j$. Hence the equivalent form of $(7)$ is

$$
\theta_{j} \Lambda=\left[\Lambda_{-}^{j}, \Lambda\right]
$$

Notice that (8) implies $\theta_{j}\left(u_{-1}+\frac{1}{2} u_{0}^{[1]}\right)=0$. This is analogous to the conservation of the $(n-1)^{\text {th }}$ coefficient in the ordinary Lax equations.

To rewrite SKP once more, we introduce the formal differential

$$
d=\sum_{i \geqq 1}\left(d t_{2 i} \partial / \partial t_{2 i}+d \tau_{2 j-1} \partial / \partial \tau_{2 j-1}\right)
$$

Using (6), we have

$$
\partial / \partial \tau_{2 j-1}=\theta_{2 j-1}-\sum_{k=1}^{\infty} \tau_{2 k-1} \theta_{2 j+2 k-2}
$$

and therefore (7) and (8) are equivalent to the equations

$$
d \Lambda=-\left[U_{\Lambda}{ }^{+}, \Lambda\right]+2 \sum_{j=1}^{\infty} d \tau_{2 j-1} \Lambda^{2 j}=\left[U_{\Lambda}{ }^{-}, \Lambda\right]
$$


where

$$
U_{\Lambda}^{ \pm}=\sum_{i=1}^{\infty} d t_{2 i} \Lambda_{ \pm}^{2 i}+\sum_{j=1}^{\infty} d \tau_{2 j-1}\left(\Lambda_{ \pm}^{2 j-1}-\sum_{k=1}^{\infty} \tau_{2 k-1} \Lambda_{ \pm}^{2 j+2 k-2}\right) .
$$

\subsection{The Superresidue and the Berezin Integral}

From now on we assume that $B$ is supercommutative. We set

$$
\operatorname{res}_{\theta}\left(\sum_{i \leqq m} b_{i} \theta^{i}\right)=b_{-1}
$$

We remind the reader that the Berezin integral $\int f(x, \xi) d(x, \xi)$ is well defined if $\frac{\partial}{\partial \xi} f$ as a function of $x$ has a compact support (or quickly vanishes at infinity). In this case $\int f(x, \xi) d(x, \xi)=\int\left(\frac{\partial}{\partial \xi} f\right) d x$. In particular $\int f(x, \xi) d(x, \xi)=0$ if $\partial f / \partial \xi$ $=\partial g / \partial x$, where $g$ quickly vanishes at infinity. The following lemma is an analogue of a well known fact in the theory of the $\mathrm{KdV}$ hierarchy (cf. [10, Chap. 2, Lemma 3.3]).

1.5. Lemma. a) Let $P, Q \in B\left(\left(\theta^{-1}\right)\right)$. Then there exists a universal polynomial $F=F(P, Q)$ depending on the coefficients of $P, Q$ and their $\theta$-derivatives, such that

$$
\operatorname{res}_{\theta}[P, Q]=\theta F(P, Q),
$$

b) $\int \operatorname{res}_{\theta}[P, Q] d(x, \xi)=0$ in the sense that

$$
\frac{\partial}{\partial \xi} \operatorname{res}_{\theta}[P, Q]=\frac{\partial}{\partial x}\left(1-\xi \frac{\partial}{\partial \xi}\right) F(P, Q) .
$$

1.6. Theorem on the Conservation Laws. The SKP equations for an operator $\Lambda$ imply the equations

$$
\theta_{i} R_{n}+\theta S_{i, n}=0, \quad i, n \geqq 1
$$

where

$$
R_{n}=\operatorname{res}_{\theta} \Lambda^{n}, \quad S_{i, n}=-F\left(\Lambda_{-}^{i}, \Lambda^{n}\right)
$$

$F$ being defined in Lemma 1.5 .

\subsection{The Zakharov-Shabat Formalism}

In this section we assume that the $\operatorname{ring} B$ has an appropriate topology. Then it may happen that the multiplication in $B\left(\left(\theta^{-1}\right)\right)$ can be defined by continuity on a certain subset of the doubly infinite formal series $B\left\{\left\{\theta^{-1}\right\}\right\}=\left\{\sum_{i} b_{i} \theta^{i}, i \in \mathbb{Z} \mid b_{i} \in B\right\}$. We shall show that certain identities in such a ring make it possible to construct a solution of SKP. Consider a differential form-valued operator

$$
U_{\theta}=\sum_{i=1}^{\infty} d t_{2 i} \theta^{2 i}+\sum_{j=1}^{\infty} d \tau_{2 j-1}\left(\theta^{2 j-1}-\sum_{k=1}^{\infty} \tau_{2 k-1} \theta^{2 j+2 k-2}\right)
$$


[cf. Eq. (10)]. Let $\Psi \in B\left\{\left\{\theta^{-1}\right\}\right\}, \widetilde{\Psi}=0$. Assume that the following relations are valid:

$$
\begin{gathered}
d \Psi=-U_{\theta} \psi, \\
\Psi=V^{-1} Y, V=1+\sum_{k=1}^{\infty} v_{-k} \theta^{-k}, Y=\sum_{\ell=0}^{\infty} y_{\ell} \theta^{\ell},
\end{gathered}
$$

where $\tilde{V}=\tilde{Y}=0$. The relation (11) is a system of linear differential equations with constant coefficients, and (12) is the decomposition of a Lie superalgebra element into a product of the upper and lower triangular parts, or a solution of the formal Riemann-Hilbert problem.

1.8. Theorem. If $Y$ is invertible in $B\left\{\left\{\theta^{-1}\right\}\right\}$ and verifies $\left(Y^{-1}\right)_{-}=0$, then $\Lambda=V \theta V^{-1}$ is a solution of $S K P$.

We point out that the "dressed" operator $\Lambda$ verifies the relation $u_{-1}+\frac{1}{2} u_{0}^{[1]}=0$.

Our next results concern the reductions of SKP to the Lax-Gelfand-Dikii hierarchies $[9,10]$.

1.9. Theorem on the Fractional Powers. Let $L=\theta^{N}+\sum_{n \leqq N-1} u_{n} \theta^{n}$. If $N>0$, $N \equiv 1(2)$, then there exists a unique odd operator $\Lambda=\theta+\sum_{i \leqq 0} v_{i} \theta^{i}$ such that $\Lambda^{N}=L$.

For $N \equiv 0 \bmod 2$ both existence and uniqueness need not be true.

\subsection{Variational Formalism}

In the following we shall consider the coefficients $u_{i}$ of the differential operator $L$ as differentially independent variables. To this end we set $B=A\left[u_{i}^{[j]}\right], \tilde{u}_{i}^{[j]}=\tilde{u}_{i}+\tilde{j}$, where $u_{i}^{[j]}$ are algebraically independent variables and $A$ is a supercommutative ring with an action of $\theta$. We shall extend the action of $\theta$ to $B$, setting $\bar{\theta} \mid A=\theta$, $\bar{\theta} u_{j}^{[i]}=u_{j}{ }^{[i+1]}$, and in what follows we shall denote $\bar{\theta}$ again by $\theta$. The EulerLagrange operators are defined by the formula

$$
\frac{\delta P}{\delta u_{i}}=\sum_{k}(-1)^{\tilde{u}_{k} k+\frac{k(k+1)}{2}}\left(\frac{\partial P}{\partial u_{i}^{[k]}}\right)^{[k]} .
$$

Furthermore, we introduce the supersymmetric Gelfand-Dikii operator (cf. $[9,10])$,

$$
\begin{gathered}
\gamma=\Gamma-\Gamma^{*}, \\
\Gamma_{b c}=\sum_{a \geqq 0}(-1)^{(c+1)(b+1)}\left[\begin{array}{c}
a+b \\
b
\end{array}\right] u_{a+b+c+1} \theta^{a} .
\end{gathered}
$$

Here $\Gamma^{*}$ is defined from the adjointness property

$$
\sum_{b, c} \int\left(\Gamma_{b c} x_{c}\right) y_{b} d(x, \xi)=\sum_{b, c} \int\left(\Gamma_{b c}^{*} y_{c}\right) x_{b} d(x, \xi),
$$

where $\tilde{x}_{i}=\tilde{y}_{i}=\tilde{u}_{i}=\tilde{i}+1$. See (21) for the explicit formula. 
The operator $\gamma$ is a highly formal object, having an infinite order and infinite matrix coefficients, but it becomes finite if we set $u_{N}=1, u_{i}=0$ for $i \geqq N+1$. Denote the result by $\gamma^{(N)}$.

1.11. Theorem on the Lax Equations. Let $N \equiv p \equiv 1 \bmod 2, p \geqq N, L=\theta^{N}$ $+\sum_{n \leqq N-1} u_{n} \theta^{n}, \tilde{\Lambda}=1, \Lambda^{N}=L, \Lambda=\theta+\sum_{i \leqq 0} v_{i} \theta^{i}$. Moreover, let

$$
H\left(\frac{p}{N}\right)=\frac{N}{p} \operatorname{res}_{\theta} \Lambda^{p}
$$

Then the SKP equations for $\Lambda$ imply the Gelfand-Dikii equations for the coefficients of $L$ :

$$
\theta_{p-N} u_{b}=-\sum_{c} \gamma_{b c}^{(N)} \frac{\delta}{\delta u_{c}} H\left(\frac{p}{N}\right)
$$

\section{The Proofs}

\subsection{Some Identities with Superbinomial Coefficients}

a) The coefficients $\left[\begin{array}{l}j \\ k\end{array}\right]$ for $0 \leqq j, k \leqq 7$ constitute the matrix:

\begin{tabular}{l|llllllll}
$j$ & 0 & 1 & 2 & 3 & 4 & 5 & 6 & 7 \\
\hline 0 & 1 & & & & & & & \\
1 & 1 & 1 & & & & & & \\
2 & 1 & 0 & 1 & & & & & \\
3 & 1 & 1 & 1 & 1 & & & & \\
4 & 1 & 0 & 2 & 0 & 1 & & & \\
5 & 1 & 1 & 2 & 2 & 1 & 1 & & \\
6 & 1 & 0 & 3 & 0 & 3 & 0 & 1 & \\
7 & 1 & 1 & 3 & 3 & 3 & 3 & 1 & 1
\end{tabular}

It is clear how to continue it further. The identity $(-1)^{k} \cdot\left(\begin{array}{l}m \\ k\end{array}\right) \cdot k=(-1)^{m}\left(\begin{array}{l}-k \\ -m\end{array}\right) \cdot m$ (which follows from the defi-
nition) implies

$$
\left[\begin{array}{c}
m \\
-l-1
\end{array}\right]=(-1)^{\left[\frac{m}{2}\right]+\left[\frac{\ell}{2}\right]+1}\left[\begin{array}{c}
\ell \\
-m-1
\end{array}\right] \text { for } \ell, m \in \mathbb{Z} .
$$

b) The most direct way to describe multiplication in $B\left(\left(\theta^{-1}\right)\right)$ is to consider (4) as the definition. Then it is necessary to prove the associativity law: $(P \circ Q) \circ R=P \circ(Q \circ R)$. Collecting together similar terms we can reduce it to a bilinear identity in $\left[\begin{array}{l}j \\ k\end{array}\right]$, and then prove it directly. Another way is to use the 
well-known construction of multiplication in $B\left(\left(\partial^{-1}\right)\right)$ by means of the ordinary binomial coefficients and the fact that $B\left(\left(\theta^{-1}\right)\right)=B\left(\left(\partial^{-1}\right)\right)[\theta]$ with evident commutation relations,

$$
\theta\left(a \partial^{m}\right)=a^{[1]} \theta^{2 m}+(-1)^{\tilde{a}} a \theta^{2 m+1} .
$$

\subsection{Proof of Lemma 1.5}

The statement b) follows from a) because $\frac{\partial}{\partial \xi} \theta F(P, Q)=\frac{\partial}{\partial x}\left(1-\xi \frac{\partial}{\partial \xi}\right) F(P, Q)$.

To prove a) it is sufficient to consider the case $P=a \theta^{m}, Q=b \theta^{\ell}$. From (4) we find

Hence

$$
a \theta^{m} \circ b \theta^{\ell}=a \sum_{k \leqq m}\left[\begin{array}{l}
m \\
k
\end{array}\right](-1)^{\tilde{b} k} b^{[m-k]} \theta^{k+\ell} .
$$

$$
\operatorname{res}_{\theta}\left(a \theta^{m} \circ b \theta^{\ell}\right)=\left[\begin{array}{c}
m \\
-\ell-1
\end{array}\right](-1)^{\tilde{b}(\ell+1)} a b^{[m+\ell+1]} .
$$

By the symmetry

$$
\operatorname{res}_{\theta}\left(b \theta^{\ell} \circ a \theta^{m}\right)=\left[\begin{array}{c}
\ell \\
-m-1
\end{array}\right](-1)^{\tilde{a}(m+1)} b a^{[m+\ell+1]} .
$$

Using these two formulas and (16) we find

$$
\begin{gathered}
\operatorname{res}_{\theta}[P, Q]=\left[\begin{array}{c}
m \\
-\ell-1
\end{array}\right](-1)^{\tilde{b}(\ell+1)}\left(a b^{[m+\ell+1}+(-1)^{M} a^{[m+\ell+1]} b\right), \\
M=\tilde{a}(m+\ell+1)+m \ell+\left[\frac{m}{2}\right]+\left[\frac{\ell}{2}\right] .
\end{gathered}
$$

The right-hand side of (17) is represented as the complete $\theta$-derivative in two different ways depending on the parity of $m+\ell+1($ the case $(m, \ell) \equiv(0,0) \bmod 2$ needs no attention, since then $\left.\left[\begin{array}{c}m \\ -\ell-1\end{array}\right]=0\right)$ :

$$
\begin{aligned}
& a b^{[m+\ell+1]}+(-1)^{M} a^{[m+\ell+1]} b \\
& =\left\{\begin{array}{lll}
\theta\left(\sum_{i=0}^{m+\ell}(-1)^{\tilde{a}(i+1)+\left[\frac{i}{2}\right]} a^{[i]} b^{[m+\ell-i]}\right) & \text { if } & m+\ell+1 \equiv 1 \bmod 2 \\
\theta^{2}\left(\frac{m+\ell-1}{\sum_{i=0}^{2}}(-1)^{i} a^{[2 i]} b^{[m+\ell-2 i-1]}\right) & \text { if } & m+\ell+1 \equiv 0 \bmod 2 .
\end{array}\right.
\end{aligned}
$$

\subsection{Proof of Lemma 1.6}

From (8) we find $\theta_{i} \Lambda^{n}=\left[\Lambda^{i}{ }_{-}, \Lambda^{n}\right]$. Therefore

$$
\theta_{i} \operatorname{res}_{\theta} \Lambda^{n}=\operatorname{res}_{\theta} \theta_{i} \Lambda^{n}=\operatorname{res}_{\theta}\left[\Lambda^{i}{ }_{-}, \Lambda^{n}\right]=\theta F\left(\Lambda_{-}^{i}, \Lambda^{n}\right) .
$$




\subsection{Proof of Theorem 1.8}

In the notation of Sect. 1.7 we construct the following operators from the data (11) and (12):

$$
\Lambda=V \circ \theta \circ V^{-1}, U^{+}=(d Y) \circ Y^{-1}, U^{-}=-(d V) \circ V^{-1} .
$$

On the other hand, we construct from $\Lambda$ "connection forms" $U_{A}^{ \pm}$by the formula (10). We shall check that if the condition of Theorem 1.8 is fulfilled, then $U^{ \pm}=-U_{A}^{ \pm}$. Really, using that $\Psi$ is invertible in view of (12), we find from (11),

$$
\begin{aligned}
-U_{\theta} & =d \Psi \circ \Psi^{-1}=d\left(V^{-1} \circ Y\right) \circ Y^{-1} \circ V \\
& =\left(-V^{-1} d V \circ V^{-1} \circ Y+V^{-1} \circ d Y\right) Y^{-1} \circ V \\
& =V^{-1}\left(-d V \circ V^{-1}+d Y \circ Y^{-1}\right) V=V^{-1}\left(U^{-}+U^{+}\right) V .
\end{aligned}
$$

Hence

$$
U^{+}+U^{-}=-V U_{\theta} V^{-1}=-\sum_{i=1}^{\infty} d t_{2 i} \Lambda^{2 i}+\sum_{j=1}^{\infty} d \tau_{2 j-1}\left(\Lambda^{2 j-1}-\sum_{k=1}^{\infty} \tau_{2 k-1} \Lambda^{2 j+2 k-2}\right)
$$

and, consequently, $U^{ \pm}=-U_{A}^{ \pm}$, because $U^{+}$is a differential operator, and $U^{-}$ is an integral one. On the other hand, by the definition of $\Lambda$,

$$
\begin{aligned}
d \Lambda & =d\left(V \circ \theta \circ V^{-1}\right)=d V \circ \theta \circ V^{-1}+V \circ \theta \circ V^{-1} \circ d V \circ V^{-1} \\
& =\left[d V \circ V^{-1}, V \circ \theta \circ V^{-1}\right]=-\left[U^{-}, \Lambda\right]=\left[U_{\Lambda}^{-}, \Lambda\right],
\end{aligned}
$$

i.e. the SKP hierarchy in the form (9).

\subsection{Proof of Theorem 1.9}

Let us construct $\Lambda$ using successive approximations. Assume that at the $r^{\text {th }}$ step, $r \geqq-1$, we have proved the following statement:

there exists an operator $X_{r}=\theta+O(1)$ such, that $X_{r}^{N}=L+O\left(\theta^{N-r-2}\right)$; it is defined uniquely $\bmod O\left(\theta^{-(r+1)}\right)$.

For $r=-1$, evidently, $X_{r}=\theta$. To make the inductive step we set $X_{r+1}=X_{r}$ $+x_{r+1} \theta^{-(r+1)}$. Then

$$
X_{r+1}^{N}=X_{r}^{N}+\sum_{i=0}^{N-1} X_{r}^{i} \circ x_{r+1} \theta^{-(r+1)} \circ X_{r}^{N-i-1}+R_{r}
$$

Now we compute the right-hand side sum modulo $O\left(\theta^{N-r-3}\right)$ :

$$
\begin{aligned}
\sum & =\sum_{i=0}^{N-1}(-1)^{i} x_{r+1} \theta^{-(r+1)} \circ X_{r}^{N-1} \bmod O\left(\theta^{N-r-3}\right) \\
& =x_{r+1} \theta^{N-r-2} \bmod O\left(\theta^{N-r-3}\right) .
\end{aligned}
$$

(We use the fact that $N$ is odd only in this place: if $N$ is even the linear term in $x_{r+1}$ disappears.) The remainder is the sum of the products of $j \leqq N-2$ terms $X_{r}$ and of $N-j$ terms $x_{r+1} \theta^{-(r+1)}$. Therefore its order with respect to $\theta$ is not more than 
$\max _{j \leqq N-2}[j-(N-j)(r+1)]=N-2 r-4 \leqq N-r-3$. Hence $x_{r+1}$ is defined uniquely from the condition $X_{r+1}^{N}=L+O\left(\theta^{N-2-3}\right)$. To finish the proof we set $\Lambda=\lim _{r \rightarrow \infty} X_{r}$.

We give two examples concerning even $N$.

a) Let $L=\theta^{2 n}+\sum_{i \leq 2 n-1} u_{i} \theta^{i}, \tilde{L}=0$. If $\operatorname{res}_{\theta} L \notin \theta B$, then $L \neq \Lambda^{2 n}$ for any odd $\Lambda$. In fact, $\Lambda^{2 n}=\frac{1}{2}\left[\Lambda^{2 n-1}, \Lambda\right]$, so $\operatorname{res}_{\theta} \Lambda^{2 n}$ should be a $\theta$-derivative in view of Lemma 1.5 .

b) In the case $L=\theta^{2}$ the following nonuniqueness of the square root arises: we can take $\Lambda$ in the form $\theta+u_{0}-\frac{1}{2} u_{0}^{[1]} \theta^{-1}++\sum_{i \leqq-2} u_{i} \theta^{i}$, where the $u_{i}$ satisfy the
following conditions:

$$
\left\{\begin{array}{l}
u_{i, x}=0 \\
u_{2 p-1, \xi}=0, \\
u_{2 p, \xi}+2 u_{2 p-1}+\sum_{\ell+m=p+1}\left(u_{2 \ell-1} u_{2 m, \xi}+u_{2 \ell-1} u_{2 m-1}\right)=0
\end{array}\right.
$$

It is possible, for instance, to choose arbitrary coefficients $u_{2 p}$ independent of $x$ and then to compute $u_{2 p-1}$ by induction. In fact, the first series of equations follows from the equality

$$
0=\left[\Lambda^{2}, \Lambda\right]=\left[\partial_{x}, \Lambda\right]=\sum_{i \leq 0} u_{i, x} \theta^{i}
$$

Coefficients of $\frac{1}{2}[\Lambda, \Lambda]$ at odd powers of $\theta$ give us the following equations:

$$
u_{2 p-1, \xi}+\sum_{m+n=p+1} u_{2 m-1} u_{2 n-1, \xi}=0 \text {. }
$$

Now, inductively we get $u_{2 p-1, \xi}=0$. Finally, the coefficients at $\theta^{2 \ell}, \ell \leqq 0$, lead to the last series of equations.

\subsection{More on the Variational Formalism}

Using the notations from Sect. 1.10 we consider the $B$-module of relative differentials $\Omega=\Omega^{1} B / A$. It is freely generated by the elements $\delta u_{i}^{[j]}$. We assume that $\tilde{\delta}=0$ (that is $\delta \tilde{P}=\tilde{P})$. The derivation $\theta$ acts on $\Omega$ by the formula $\theta(\delta P)=\delta\left(P^{[1]}\right)$. Therefore we can define a bimodule $\Omega\left(\left(\theta^{-1}\right)\right)$ over the ring $B\left(\left(\theta^{-1}\right)\right)$ using the formula (4) for the exterior multiplication. The associativity axiom is verified by repeating the reasoning from Sect. 2.1b). The $\operatorname{map} \delta: B\left(\left(\theta^{-1}\right)\right) \rightarrow \Omega\left(\left(\theta^{-1}\right)\right)$ is defined termwise. It shares the property $\delta(P \circ Q)=\delta P \circ Q+P \circ \delta Q$.

Let $\omega \in \Omega$ be a variational differential. It has a unique representation in the form $\omega=\omega_{0}+\theta \omega_{1}$, where $\omega_{0} \in \oplus B \delta u_{i}$. The existence is proved by the classical procedure of integrating by parts

$$
a \delta u_{i}^{[j+1]}=(-1)^{\tilde{a}+1}\left(a^{[1]} \delta u_{i}^{[j]}-\theta\left(a \delta u_{i}^{[j]}\right)\right) .
$$

The uniqueness follows from the fact: if $\theta \omega_{1} \neq 0$, then $\theta \omega_{1} \notin \oplus B \delta u_{i}$ because the term $a \delta u_{i}^{[j]}$ in $\omega_{1}$ with maximal $j$ gives the term $a \delta u_{i}^{[j+1]}$ in $\theta \omega_{1}$ which cannot cancel. 
It is easy to derive from (18) the following lemma, giving the invariant description of the Euler-Lagrange operators (13):

2.7. Lemma. Let $P \in B$ and $\delta P=\sum \delta u_{i} Q_{i}+\theta \omega_{1}$. Then $Q_{i}=\frac{\delta P}{\delta u_{i}}$.

To prove Theorem 1.11 we need the following facts:

2.8. Lemma. a) Let $\Lambda \in B\left(\left(\theta^{-1}\right)\right), \tilde{\Lambda}=1, \Lambda=\theta+\sum_{i \leqq 0} v_{i} \theta^{i}, p \geqq 1$. Then

$$
\delta \operatorname{res}_{\theta} \Lambda^{p} \equiv\left\{\begin{array}{lll}
p \operatorname{res}_{\theta}\left(\delta \Lambda \circ \Lambda^{p-1}\right) & \text { if } & p \equiv 1(2) \\
0 & \text { if } & p \equiv 0(2)
\end{array}\right\} \bmod \theta \Omega .
$$

b) Let $L=\Lambda^{N}$. Then

$$
\delta \operatorname{res}_{\theta} \Lambda^{p} \equiv \frac{p}{N} \operatorname{res}_{\theta}\left(\delta L \circ \Lambda^{p-N}\right) \bmod \theta \Omega \quad \text { if } \quad N \equiv p \equiv 1 \bmod 2 .
$$

Proof. a) We have

$$
\begin{aligned}
& \delta \Lambda^{p}=\sum_{i=0}^{p-1} \Lambda^{i} \circ \delta \Lambda \circ \Lambda^{p-i-1}, \\
& \Lambda^{i} \circ \delta \Lambda \circ \Lambda^{p-i-1}=(-1)^{i(p-i)}\left(\delta \Lambda \circ \Lambda^{p-1}-\left[\delta \Lambda \circ \Lambda^{p-i-1}, \Lambda^{i}\right]\right) .
\end{aligned}
$$

Furthermore $\sum_{i=0}^{p-1}(-1)^{i(p-i)}=p$ if $p \equiv 1(2)$ and 0 if $p \equiv 0(2)$. So the statement a) follows from Lemma 1.5, remaining undoubtedly valid when it is applied to commutators in

$$
\left[\Omega\left(\left(\theta^{-1}\right)\right), B\left(\left(\theta^{-1}\right)\right)\right]
$$

b) Similarly

$$
\delta \Lambda^{N} \circ \Lambda^{p-N}=\sum_{i=0}^{N-1} \Lambda^{i} \circ \delta \Lambda \circ \Lambda^{p-i-1} \equiv N \delta \Lambda \circ \Lambda^{p-1} \bmod \theta \Omega \quad \text { if } \quad N \equiv 1(2) .
$$

This finishes the proof.

2.9. Lemma. Let $N \equiv 1(2), L=\theta^{N}+\sum_{i=0}^{N-1} u_{i} \theta^{i}, \tilde{L}=1, u_{i}$ being independent differential variables. Let $\Lambda$ be the odd $N^{\text {th }}$ power root from $L$, constructed in Sect. 2.5. We define the differential polynomials $v_{k}\left(\frac{p}{N}\right)$ in $u_{i}$ by the formula

$$
\Lambda_{-}^{p}=\left(L^{p / N}\right)_{-}=\sum_{k \leqq 0} \theta^{k-1} \circ v_{k}\left(\frac{p}{N}\right) .
$$

Then if $p \equiv 1 \bmod 2$, we have for $k \leqq N-1$

$$
\frac{\delta}{\delta u_{k}} v_{0}\left(\frac{p}{N}\right)=\frac{p}{N}(-1)^{k+1} v_{-k}\left(\frac{p-N}{N}\right) .
$$


Proof. Since $v_{0}\left(\frac{p}{N}\right)$ is even, $\operatorname{res}_{\theta} \Lambda^{p}=v_{0}\left(\frac{p}{N}\right)$. From the previous lemma we have

$$
\begin{aligned}
\delta v_{0}\left(\frac{p}{N}\right) & =\delta \operatorname{res}_{\theta} \Lambda^{p} \equiv \frac{p}{N} \operatorname{res}_{\theta}\left(\delta L \circ \Lambda^{p-N}\right) \\
& =\frac{p}{N} \operatorname{res}_{\theta}\left(\sum_{j=0}^{N-1} \delta u_{j} \theta^{j} \circ \sum_{k \leqq 0} \theta^{k-1} \circ v_{k}\left(\frac{p-N}{N}\right)\right) \\
& =\frac{p}{N} \operatorname{res}_{\theta}\left(\sum_{j=0}^{N-1} \delta u_{j} \theta^{-1} \circ v_{-j}\left(\frac{p-N}{N}\right)\right) \\
& =\frac{p}{N} \sum_{j=0}^{N-1} \delta u_{j}(-1)^{j+1} v_{-j}\left(\frac{p-N}{N}\right) .
\end{aligned}
$$

To finish the proof we use Lemma 2.6.

\subsection{The End of the Proof of Theorem 1.11}

From the SKP equations for $\Lambda$ we derive the following equations for $L$ :

$$
-\theta_{p-N} L=\left[L, \Lambda^{p-N}\right]
$$

or

$$
-\sum_{b=0}^{N-1}\left(\theta_{p-N} u_{b}\right) \theta^{b}=\left[\sum_{\ell=0}^{N} u_{\ell} \theta^{\ell}, \sum_{j \geqq 0} \theta^{-j-1} \circ v_{-j}\left(\frac{p-N}{N}\right)\right] .
$$

We shall show that the coefficient at $\theta^{b}$ in the first member of the commutator is equal to $\sum_{c} \Gamma_{b c} \frac{\delta}{\delta u_{c}} v_{0}\left(\frac{p}{N}\right)$, where $\Gamma_{b c}$ are defined in (14). Really

$$
\left[L, \Lambda^{p-N}\right]=-\left[L, \Lambda^{p-N}+\right]=\left[L, \Lambda^{p-N}\right]_{+} .
$$

Hence it is sufficient to compute $\left(L \Lambda^{p-N_{-}}\right)_{+}$and $\left(\Lambda^{p-N}{ }_{-} L\right)_{+}$. Using Lemma 2.9 we have

$$
\begin{aligned}
\left(L \Lambda^{p-N}\right)_{+}=\left(\sum_{\ell=0}^{N} u_{\ell} \theta^{\ell} \circ \sum_{j \geqq 0}(-1)^{j+1} \theta^{-(j+1)} \frac{\delta}{\delta u_{j}} H\left(\frac{p}{N}\right)\right)_{+} \\
=\left(\sum_{\ell=0}^{N} \sum_{j \geqq 0}(-1)^{j+1} u_{\ell} \sum_{b}\left[\begin{array}{c}
\ell-j-1 \\
b
\end{array}\right](-1)^{b(j+1)}\right. \\
\left.\cdot\left(\frac{\delta}{\delta u_{j}} H\left(\frac{p}{N}\right)\right)^{[\ell-j-1-b]} \theta^{b}\right)_{+} \\
=\sum_{\substack{a, b, c \geqq 0 \\
a+b+c+1 \leqq N}}(-1)^{(c+1)(b+1)} u_{a+b+c+1}\left[\begin{array}{c}
a+b \\
b
\end{array}\right]\left(\frac{\delta}{\delta u_{c}} H\left(\frac{p}{N}\right)\right)^{[a]} \theta^{b} .
\end{aligned}
$$


Here we have denoted $\ell-j-b-1=a, j=c$. Computing $\left(\Lambda^{p-N}{ }_{-} L\right)_{+}$, we obtain at first

$$
\sum_{\substack{a, b, c \geqq 0 \\
a+b+c+1 \leqq N}}\left[\begin{array}{c}
-c-1 \\
-a-c-1
\end{array}\right](-1)^{a b}\left(u_{a+b+c+1} \frac{\delta}{\delta u_{c}} H\left(\frac{p}{N}\right)\right)^{[a]} \theta^{b} .
$$

Then we transform the superbinomial coefficient using (16):

$$
\left[\begin{array}{c}
-c-1 \\
-a-c-1
\end{array}\right]=(-1)^{\left[\frac{-c-1}{2}\right]+\left[\frac{a+c}{2}\right]+1}\left[\begin{array}{c}
a+c \\
c
\end{array}\right]
$$

If $a$ or $c$ are even, then the sign is equal to $(-1)^{\left[\frac{a}{2}\right]}$. If $a$ and $c$ are both odd then the coefficients vanish. Finally

$$
\left(\Lambda^{p-N}-L\right)_{+}=\sum_{\substack{a, b, c \geqq 0 \\
a+b+c+1 \leqq N}}(-1)^{\left[\frac{a}{2}\right]+a b}\left[\begin{array}{c}
a+c \\
c
\end{array}\right]\left(u_{a+b+c+1} \frac{\delta}{\delta u_{c}} H\left(\frac{p}{N}\right)\right)^{[a]} \circ \theta^{b} .
$$

The reader can check that the operators we have computed are adjoint.

2.11. Examples. Set $L=\Lambda^{4}=\Lambda_{+}^{4}=\theta^{4}+v_{1} \theta+v_{0}, \quad \tilde{v}_{1}=1, \quad \tilde{v}_{0}=0$. Then Lax's equation $L_{t}=\left[L_{+}^{3 / 2}, L\right]$ is equivalent to

$$
\left.\begin{array}{l}
v_{0 t}=\partial_{x}\left(v_{0 x x} / 4+3 v_{0}^{2} / 4+3 v_{1} v_{0}^{[1]} / 4\right) \\
v_{1 t}=\partial_{x}\left(v_{1 x x} / 4+3 v_{1} v_{1}^{[1]} / 4+3 v_{1} v_{0} / 2\right)
\end{array}\right\}
$$

Setting here $v_{1}=0$ we get $\mathrm{KdV}$. On the other hand, setting $v_{0}=0$ and $v_{1}=w_{1}+\xi w_{0}$, $\tilde{w}_{1}=1, \tilde{w}_{0}=0$, we get from $(22)$

$$
\left.\begin{array}{l}
w_{0 t}=\partial_{x}\left(w_{0 x x} / 4+3 w_{0}^{2} / 4-3 w_{1} w_{1 x} / 4\right) \\
w_{1 t}=\partial_{x}\left(w_{1 x x} / 4+3 w_{0} w_{1} / 4\right)
\end{array}\right\}
$$

Setting $w_{1}=0$ here we again get $\mathrm{KdV}$. The first Eq. (23) after rescaling coincides with the first equation of Kupershmidt's SKdV, but the second equations are different (cf. [3]).

\section{References}

1. Saveliev, M.: Integrable supermanifolds and nonlinear systems. Preprint IHEP 84-20, Serpukhov (1984)

2. Chaichian, M., Kulish, P.: On the method of inverse scattering problem and Bäcklund transformations for supersymmetric equations. Phys. Lett. 78 B, 413 (1978)

3. Kupershmidt, B.: A super-Korteweg-deVries equation: an integrable system. Preprint UTSI, Tullahoma (1984)

4. d'Auria, R., Sciuto, S.: Group theoretical construction of two-dimensional supersymmetric models. Nucl. Phys. B 171, 189 (1980)

5. Date, E., Kashiwara, M., Miwa, T.: Vertex operators and $\tau$-functions. Transformation groups for soliton equations. II. Proc. Jpn. Acad. Sci. 57 A, 387 (1981) 
6. Date, E., Jimbo, M., Kashiwara, M., Miwa, T.: Operator approach to the KadomtsevPetviashvili equation. Transformation groups for soliton equations. III. J. Phys. Soc. Jpn. 50, 3866 (1981)

7. Wess, J.: Supersymmetry-supergravity. In: Lecture Notes in Physics, Vol. 77. Berlin, Heidelberg, New York: Springer 1978

8. Leites, D.: Introduction to the theory of supermanifolds. Russ. Math. Surv. 35, 3 (1980)

9. Gelfand, I., Dikii, L.: Fractional powers of operators and hamiltonian systems. Funct. Analiz 10, 13 (1976)

10. Manin, Yu.: Algebraic aspects of differential equations. J. Sov. Math. 11, 1 (1979)

Communicated by Ya. G. Sinai

Received June 15, 1984 
